\title{
BMJ Open Comparison between recent and long- term physical activity levels as predictors of cardiometabolic risk: a cohort study
}

\author{
Tuija Leskinen (D) ,,2 Sari Stenholm, ${ }^{1,2}$ Anna Pulakka (D) , 1,2,3 Jaana Pentti, ${ }^{1,4}$ \\ Mika Kivimäki, ${ }^{4,5,6}$ Jussi Vahtera (D) ${ }^{1,2}$
}

To cite: Leskinen T, Stenholm S, Pulakka A, et al. Comparison between recent and long-term physical activity levels as predictors of cardiometabolic risk: a cohort study. BMJ Open 2020;10:e033797. doi:10.1136/ bmjopen-2019-033797

- Prepublication history for this paper is available online. To view these files, please visit the journal online (http://dx.doi. org/10.1136/bmjopen-2019033797).

Received 22 August 2019 Revised 20 December 2019 Accepted 23 January 2020

Check for updates

(c) Author(s) (or their employer(s)) 2020. Re-use permitted under CC BY-NC. No commercial re-use. See rights and permissions. Published by BMJ.

${ }^{1}$ Department of Public Health, University of Turku and Turku University Hospital, Turku,

Finland

${ }^{2}$ Centre for Population Health Research, University of Turku and Turku University Hospital, Turku, Finland

${ }^{3}$ National Institute for Health and Welfare, Helsinki, Finland

${ }^{4}$ Clinicum, Faculty of Medicine, University of Helsinki, Helsinki, Finland

${ }^{5}$ Finnish Institute of Occupational Health, Helsinki, Finland

${ }^{6}$ Department of Epidemiology and Public Health, University College London, London, UK

Correspondence to

Dr Tuija Leskinen;

tuija.leskinen@utu.fi

\section{ABSTRACT}

Objective To compare recent and long-term physical activity levels as predictors of cardiometabolic risk in a risk factor-free adult population.

Design A 12-year prospective cohort study.

Setting The Finnish Public Sector study with surveys conducted in four waves at 4-year intervals.

Participants 19230 participants (mean age 50.2 (SD 9.1) years, $84 \%$ women) with no prevalent cardiometabolic risk factors at wave 3 were included. Physical activity was assessed at waves 1, 2 and 3. The long-term physical activity level was determined as the mean of activity from wave 1 to 3 (over 8 years).

Outcome measure 4-year incidence of cardiometabolic risk factors (obesity, hypertension, dyslipidaemia and diabetes) after wave 3 , measured individually and as a sum (accumulation of two or more risk factors vs none). Logistic and multinomial logistic regression analyses were used for the analysis.

Results Graded associations between higher physical activity levels and lower odds of all risk factors were observed ( $p$ for trend $<0.01$ ). In comparison with the persistently vigorously active participants ( $\geq 60$ metabolic equivalent (MET)-hours/week), those who were persistently inactive ( $<7$ MET-hours/week) were about four times more likely to develop obesity ( $\mathrm{OR}=4.24,95 \% \mathrm{Cl}=2.83$ to 6.36$)$, two times more likely to develop hypertension $(\mathrm{OR}=2.02$, $95 \% \mathrm{Cl}=1.45$ to 2.82 ) and dyslipidaemia $(\mathrm{OR}=1.82$, $95 \% \mathrm{Cl}=1.03$ to 3.22 ) and eight times more likely to develop diabetes $(\mathrm{OR}=7.84,95 \% \mathrm{Cl}=1.78$ to 34.6$)$. The corresponding $\mathrm{OR}$ for accumulating two or more risk factors was 5.24 -fold $(95 \% \mathrm{Cl}=2.39$ to 11.47$)$. For recently inactive versus recently vigorously active, the estimates were weaker $(\mathrm{OR}=2.36,95 \% \mathrm{Cl}=1.71$ to 3.25 for obesity; 1.78 , $95 \% \mathrm{Cl}=1.35$ to 2.35 for hypertension; $1.71,95 \% \mathrm{Cl}=1.04$ to 2.82 for dyslipidaemia; $3.56,95 \% \mathrm{Cl}=1.06$ to 11.96 for diabetes and $2.66,95 \% \mathrm{Cl}=1.48$ to 4.78 for $\geq 2$ risk factors). Conclusion Cardiometabolic risk associated with physical inactivity is better captured by repeated measurements of physical activity than by a single measurement of the most recent activity level.

\section{INTRODUCTION}

Physical inactivity is among the leading risk factors for the burden of disease, ranking
Strengths and limitations of this study

Finnish Public Sector study with repeated measurements of physical activity over 8 years.

- A large sample of risk factor-free adult population.

- Survey data linked to the records of national health registers to ascertain incident hypertension, dyslipidaemia and diabetes.

- Limitations include the self-reported assessments of physical activity and body mass index.

- Lack of data on total physical activity, sedentary time, dietary intake and metabolic traits.

at the 14th position globally and at the 11th position in high-income countries. ${ }^{1}$ Physical inactivity increases the risk of cardiometabolic diseases, such as diabetes, coronary heart disease and stroke, while also contributing to other health outcomes, such as specific cancers and mortality. ${ }^{2-5}$ Randomised controlled trials have shown cardiometabolic benefits of physical activity interventions even with short-term follow-up periods. ${ }^{6}$ Prospective observational studies have reported a lower health risk in physically active individuals as compared with inactive individuals. ${ }^{7}$ These studies have typically relied on a single assessment of physical activity, which does not take into account that physical activity may change over time. ${ }^{8}$ To date, very few studies with repeated measurements of physical activity in healthy population are available. ${ }^{9}$ Thus, the effect of long-term physical activity for the prevention of cardiometabolic risk remains uncertain.

In this study with a risk factor-free adult population, we examined whether the long-term physical activity level based on repeated measurements over an 8-year period was associated with subsequent incidence and accumulation of obesity, hypertension, dyslipidaemia and diabetes more 
strongly than the physical activity level based on a single measurement.

\section{METHODS}

\section{Study population}

The study population consisted of participants of the Finnish Public Sector (FPS) study, an ongoing open cohort study on employees in 10 towns and 6 hospital districts in Finland, including all those who were employed for a minimum of 6 months between the years 1997 and $2005 .{ }^{10}$ The FPS participants represent a wide range of occupations from semiskilled cleaners to physicians and mayors. The material has been collected through surveys at 4-year intervals since 1997/1998. Survey questionnaires were sent to all current employees of the target organisations as well as those who had participated in the earlier surveys but were no longer employed by the relevant organisation. Responding to the survey was always voluntary, and, by responding, the cohort members gave a permission for their responses to be linked to the national health registers. The participants of the current study had responded to the surveys in 1997/1998 (wave 1), 2000/2002 (wave 2), 2004/2005 (wave 3) and 2008/2009 (wave 4), or in 2000/2002 (wave 1), 2004/2005 (wave 2), 2008/2009 (wave 3) and 2012/2013 (wave 4). For the analyses, all data were pseudonymised, and researchers had only access to data with research identifications.

Of the participants who had responded at wave 1 $(\mathrm{n}=53416,71 \%$ from the eligible population), 32004 also responded at waves 3 and 4 . Those who responded at waves 3 and 4 were older (44.9 (SD 9.1) vs 42.3 (SD 9.8 ) years), more often women ( $83 \%$ vs $78 \%$ ) and highly educated (57\% vs $49 \%)$ at wave 1 than those who did not respond at waves 3 and $4(\mathrm{n}=18339)$. We excluded from the analysis participants who had missing information on physical activity at waves 1 and $3(\mathrm{n}=575)$, missing information on risk factors at waves 3 and $4(\mathrm{n}=2164)$, history of cardiovascular events at wave $3(\mathrm{n}=494)$ and $\geq 1$ risk factors at wave $3(n=9541)$. The final sample included 19230 risk factor-free participants (mean age 50.2 (SD 9.1) years, $84 \%$ women). For details of the sample selection, see figure 1 . In terms of age, sex and education, the cohort with wave 1 in 1997/1998 ( $\mathrm{n}=6303$, mean age 50.1 (SD 8.7) years, $87 \%$ women, $60 \%$ high education) did not differ from the cohort with wave 1 in 2000/2002 $(\mathrm{n}=12927$, mean age 50.3 (SD 9.2) years, $83 \%$ women, $61 \%$ high education).

\section{Assessment of physical activity}

Physical activity was assessed repeatedly at waves 1, 2 and 3 . The respondents were asked to estimate their average weekly hours of both leisure time and commuting physical activity during the past year using four different intensity levels: walking, brisk walking, jogging and running (or other activities with a corresponding intensity level). Each intensity level had five response alternatives, and the class midpoints were used to calculate the time spent for each

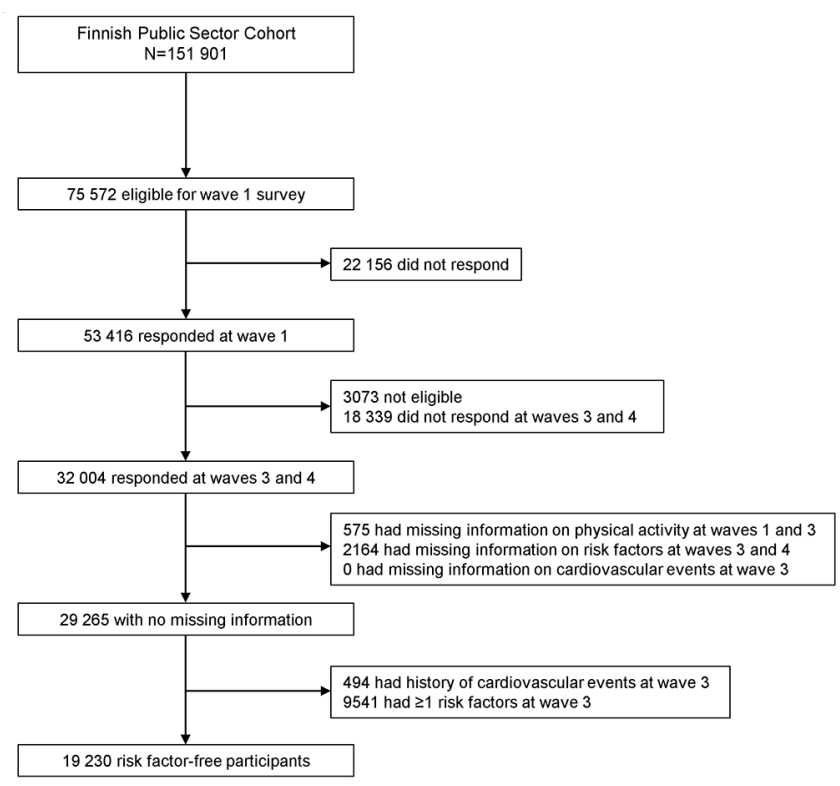

Figure 1 The selection of the sample for analysis.

intensity level: no activity ( $0 \mathrm{~min}$ used for calculation), less than 0.5 hours $(15 \mathrm{~min}), \sim 1$ hour ( $45 \mathrm{~min}), 2-3$ hours (2.5 hour) and $\geq 4$ hours/week (5 hour). ${ }^{11}$ The time spent on physical activity at each intensity level, given in hours per week, was multiplied by the average energy expenditure of each activity $(3.5,5,8$ and 11 metabolic equivalents (METs) for walking, brisk walking, jogging and running, respectively). The volume of physical activity at each study wave was quantified as MET-hours/week. ${ }^{12}$

The participants were categorised into five physical activity categories by (1) the volume of physical activity reported at wave 3 (recent physical activity level) and (2) by the mean level of physical activity across waves 1-3 (long-term physical activity level). The five categories were as follows: inactive ( $<7$ MET-hours/week), low ( $\geq 7-<14$ MET-hours/week), moderate $(\geq 14-<30$ METhours/week), high ( $\geq 30-<60$ MET-hours/week) and vigorous activity level ( $\geq 60$ MET-hours/week). Most of the participants $(81 \%)$ had physical activity data available from all of the three study waves.

\section{Assessment of cardiometabolic risk factors}

For the cardiometabolic risk factors examined as outcomes, data on hypertension, dyslipidaemia and diabetes were obtained from the national health registers. To identify cases of hypertension, dyslipidaemia and diabetes, we used data on prescription purchases of antihypertensive medication, lipid-lowering medication (statins) and antidiabetic medication, respectively, as described previously. ${ }^{13}$ Those participants who had become eligible for special reimbursement for antihypertensive or diabetes treatment, or who had purchased antihypertensive, lipid-lowering or diabetes medication for a minimum of 3 months' dose for the first time after wave 3 , were considered as incident cases of hypertension, dyslipidaemia or diabetes. ${ }^{13}$ Incident obesity was defined as 
body mass index $(\mathrm{BMI}) \geq 30 \mathrm{~kg} / \mathrm{m}^{2}$ based on self-reported body weight and height.

Accumulation of risk factors was calculated for each participant at wave 4 as a sum of incident hypertension, dyslipidaemia, diabetes and obesity (range from 0 to 4 ), and categorised into 0,1 and $\geq 2$ risk factors.

\section{Covariates}

All covariates were derived from wave 3. Participants' age and gender were obtained from the employers' registers. The highest educational level of the participants, obtained from Statistics Finland, was coded as low $(\leq 9$ years), intermediate ( $>9$ to $\leq 12$ years) or high ( $>12$ years). Occupation was dichotomised into non-manual (ISCO 1-4) and manual (ISCO 5-9) occupations according to the International Standard Classification of Occupations (ISCO). ${ }^{14}$ Behavioural health risks (high alcohol consumption and current smoking) were defined on the basis of the survey responses. High alcohol consumption was indicated by self-reported average consumption of more than $288 \mathrm{~g}$ /week of pure alcohol for men and $192 \mathrm{~g}$ /week for women, or having passed out due to excessive alcohol consumption at least once during the past 12 months. Self-reported smoking status was dichotomised into current smoker and current non-smoker. Information on prevalent chronic conditions $(0 / \geq 1)$, including asthma, rheumatoid arthritis, cancer or depression, was obtained from the national health registers.

\section{Patient and public involvement}

This is a secondary analysis of pre-existing datasets. No patients were involved in setting the present research question, the outcome measures or in developing the plans for recruitment, design or implementation of the study. No patients were asked to advice on the interpretation or writing up of results. The dissemination plan is targeted to a wide audience, including members of the public, patients, health professionals and experts in the specialty through various channels: written communication, events and conferences, networks and social media.

\section{Statistical analysis}

Logistic regression was used to examine the association between recent and long-term physical activity levels and subsequent 4-year incidence of the individual cardiometabolic risk factors. Multinomial logistic regression was used to examine the association between physical activity levels and accumulation of cardiometabolic risk factors, with those having none of the risk factors as the reference category. All the models were adjusted for age, sex, education, occupation, alcohol consumption, smoking status and prevalent chronic conditions at wave 3 . The SAS V.9.4 Statistical Package was used for the analyses (SAS Institute).

\section{RESULTS}

At wave 3, the mean age of the participants was 50.2 (SD 9.1) years. Of the participants, $84 \%$ were women, $61 \%$
Table 1 Characteristics of the study population at the start of the risk factor follow-up (wave 3 )

\begin{tabular}{lc}
\hline & N (\%) \\
\hline Total N & 19230 \\
Mean age (SD), years & $50.2(9.1)$ \\
Sex & \\
\hline Women & $16204(84)$ \\
\hline Men & $3026(16)$ \\
\hline Education & \\
\hline High (>12 years) & $11716(61)$ \\
\hline Intermediate & $6002(31)$ \\
\hline Low (s9 years) & $1512(8)$ \\
\hline Occupation & \\
\hline Non-manual & $12914(67)$ \\
\hline Manual & $6081(32)$ \\
\hline Physical activity level & \\
\hline Vigorous & $1561(8)$ \\
\hline High & $5901(31)$ \\
\hline Moderate & $6047(31)$ \\
\hline Low & $3188(17)$ \\
\hline Inactive & $2533(13)$ \\
\hline High alcohol consumption & \\
\hline No & $17501(91)$ \\
\hline Yes & $1686(9)$ \\
\hline Current smoking & $16750(87)$ \\
\hline No & $2151(11)$ \\
\hline Yes & \\
\hline Prevalent chronic condition* & \\
\hline No & $4167(22)$ \\
\hline Yes & \\
\hline
\end{tabular}

*Asthma, rheumatoid arthritis, cancer or depression.

had high education, $67 \%$ had non-manual occupation, 9\% had high alcohol consumption, $11 \%$ were current smokers and $22 \%$ had $\geq 1$ prevalent chronic conditions (table 1 ). At wave $3,8 \%$ of the participants reported vigorous, $31 \%$ high, $31 \%$ moderate, $17 \%$ low and $13 \%$ inactive physical activity level.

Figure 2 shows the associations between recent and long-term physical activity levels and 4-year incidence of different cardiometabolic risk factors. Overall, we found a graded relationship between higher physical activity levels and lower odds for each cardiometabolic risk factor (recent physical activity $\mathrm{p}$ for trend $\leq 0.0095$, long-term physical activity $\mathrm{p}$ for trend $\leq 0.0065$ ). Both recent and long-term physical activity levels were similarly associated with the risk of incident hypertension and dyslipidaemia: the lower the activity, the higher the risk. Similar relationships were seen for incident obesity and diabetes, but the odds were two times higher for the less active than for 


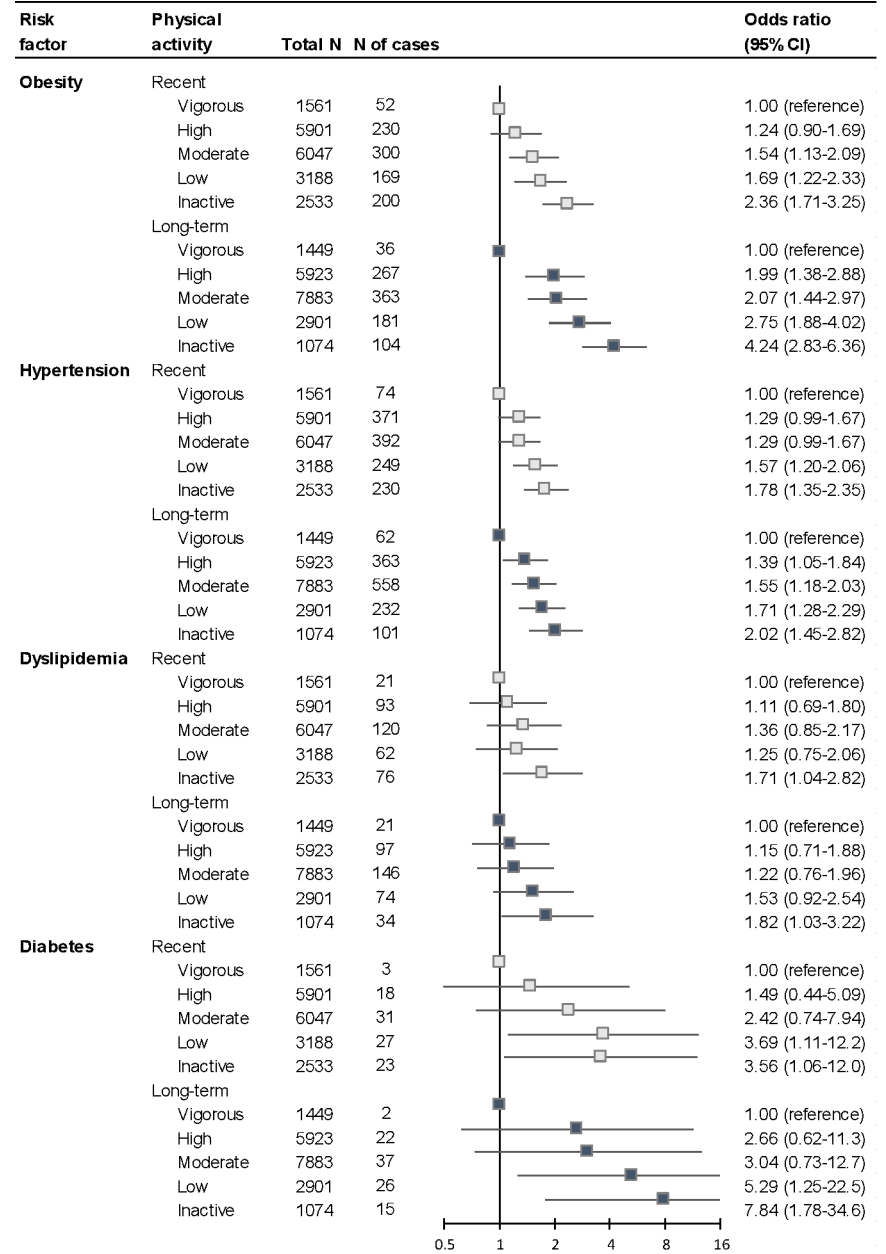

Figure 2 Association between recent and long-term physical activity levels and incident cardiometabolic risk factors. Adjusted for age, sex, education, occupation, alcohol consumption, smoking and prevalent chronic conditions at wave 3.

the vigorously active when using the long-term physical activity level as the exposure.

The association between recent and long-term physical activity levels and accumulation of risk factors is illustrated in figure 3 . The graded association for developing one risk factor versus none ( 1 vs 0 ) was similar for recent and long-term physical activity, the odds being highest for the inactive. The odds for accumulating more than one ( $\geq 2$ vs 0 ) risk factors were higher for the long-term physical activity. For example, as compared with the vigorously active participants, the risk of having $\geq 2$ risk factors was 2.7 times higher for the recently inactive and 5.2 times higher for persistently inactive individuals.

\section{DISCUSSION}

In this prospective cohort study based on repeated measurements of physical activity over 8 years, we observed a graded relationship between higher physical activity levels and lower incidence of subsequent cardiometabolic risk factors, namely obesity, hypertension, dyslipidaemia

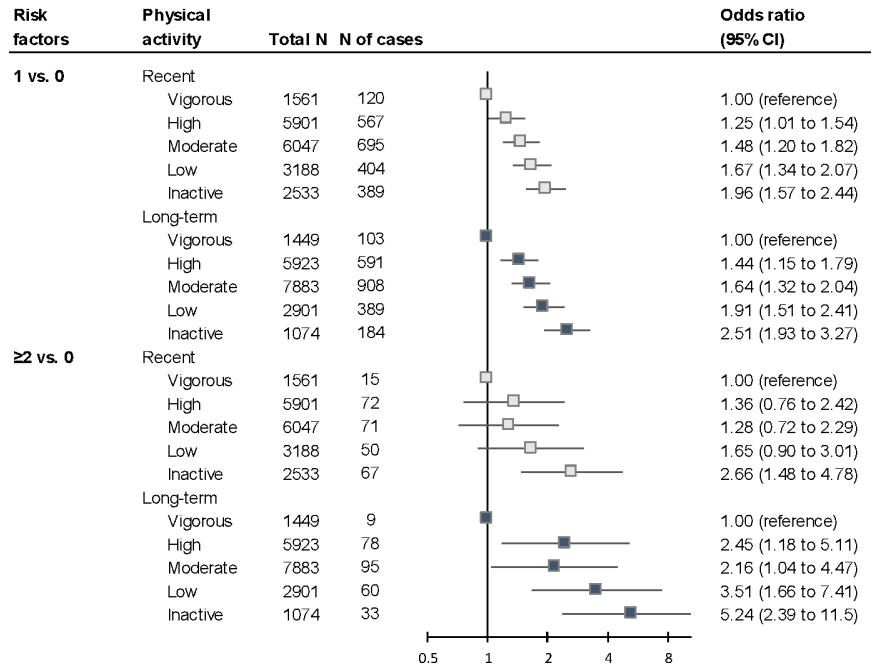

Figure 3 Association between recent and long-term physical activity levels and accumulation of cardiometabolic risk factors. Adjusted for age, sex, education, occupation, alcohol consumption, smoking and prevalent chronic conditions at wave 3 .

and diabetes. These associations were substantially stronger for long-term physical activity level as compared with a single measurement of recent physical activity level.

Our results corroborate previous findings showing that engagement in regular physical activity reduces the risk of cardiometabolic risk factors. ${ }^{15-18}$ Our findings also strengthen the evidence of an inverse dose-response relationship between the level of physical activity and cardiometabolic risk. ${ }^{719}$ Furthermore, our study adds new evidence to support the superiority of using repeated measurements of physical activity rather than a single most recent measurement of physical activity for prediction of future cardiometabolic risk, especially among risk factor-free but less active adults. Those who were persistently inactive were at the highest risk for developing and accumulating cardiometabolic risk factors, which further highlights the health burden related to physical inactivity. ${ }^{4}$

The strengths of this study include the repeated measurements of physical activity in adult population and the large number of risk factor-free participants included in the analysis. Data on all cardiometabolic risk factors, except for obesity, were obtained from reliable national health registers, and the risk factors were explored both individually and for their accumulation. It was not possible to differentiate cases of type 1 and 2 diabetes from the prescription purchase data, but any new cases of diabetes in an adult population at the average age of 50 are likely to be of type 2 . Metabolic data were not available for this study, which precludes comparisons of adiposity, glycaemic traits or systolic and diastolic blood pressure between physically active and inactive participants. Since BMI was based on self-reported values, it was likely to be underestimated, which is a weakness of this study.

There are also other limitations that should be acknowledged. The self-reported assessment of physical activity 
could be regarded as a weakness of this study, because it may be susceptible to under-reporting. ${ }^{20}$ However, the current physical activity recommendations are still grounded on studies using self-reported physical activity data, ${ }^{21}$ and it is not yet possible to obtain accelerometer measured data on physical activity over a period of 8 years with a large sample size. We had no information on workrelated physical activity and, thus, were unable to obtain an estimate of total activity during the day. Nevertheless, adjustment for occupation, a correlate of work-related physical activity, had little effect on the results, which suggests that this is not a major source of confounding. We were also not able to estimate sedentary behaviour, which is regarded as an independent risk factor for cardiometabolic diseases, at least for those with low levels of physical activity. ${ }^{7}$ The lack of dietary data is another major limitation of this study, because the cardiometabolic risk factors are strongly influenced by dietary factors. ${ }^{22} 23$ Changes in the energy intake and/or food quality during the follow-up period may have influenced our findings. Finally, the high proportions of highly educated persons and women in the study participants are in agreement with the characteristics of public sector employees in Nordic welfare settings, but they may limit the generalisability of our results beyond this population.

In conclusion, our findings suggest that cardiometabolic risk associated with physical inactivity is better captured by repeated measurements of physical activity than by a single measurement of activity level.

Contributors MK and JV had substantial contributions to the conception of the study, design of the work and acquisition of the data. TL, SS, AP and JP participated to the analysis and interpretation of data. TL drafted the manuscript, and SS, AP, JP, MK and JV revised it critically. All authors approved the final version.

Funding This study was supported by funding granted by the Juho Vainio Foundation, Finland (to TL); the Academy of Finland (Grants 286294, 294154 and 319246 to SS; 633666 to MK; 309526 to TL); the Finnish Ministry of Education and Culture (to SS); NordForsk (to MK and JV) and the UK MRC (Grant K013351 to MK).

\section{Competing interests None declared.}

Patient consent for publication Not required.

Ethics approval The Finnish Public Sector study has been approved by the Ethics Committee of the Hospital District of Helsinki and Uusimaa (HUS/1210/2016).

Provenance and peer review Not commissioned; externally peer reviewed.

Data availability statement Data are available upon reasonable request. Deidentified participant data, Finnish Public Sector Cohort.

Open access This is an open access article distributed in accordance with the Creative Commons Attribution Non Commercial (CC BY-NC 4.0) license, which permits others to distribute, remix, adapt, build upon this work non-commercially, and license their derivative works on different terms, provided the original work is properly cited, appropriate credit is given, any changes made indicated, and the use is non-commercial. See: http://creativecommons.org/licenses/by-nc/4.0/.

\section{ORCID iDs}

Tuija Leskinen http://orcid.org/0000-0001-7499-6128

Anna Pulakka http://orcid.org/0000-0002-0602-8632

Jussi Vahtera http://orcid.org/0000-0002-6036-061X

\section{REFERENCES}

1 Gakidou E, Afshin A, Abajobir AA, et al. Global, regional, and national comparative risk assessment of 84 behavioural, environmental and occupational, and metabolic risks or clusters of risks, 1990-2016: a systematic analysis for the Global Burden of Disease Study 2016. Lancet 2017;390:1345-422.

2 Li J, Siegrist J. Physical activity and risk of cardiovascular disease-a meta-analysis of prospective cohort studies. Int J Environ Res Public Health 2012;9:391-407.

$3 \mathrm{Kyu} \mathrm{HH}$, Bachman VF, Alexander LT, et al. Physical activity and risk of breast cancer, colon cancer, diabetes, ischemic heart disease, and ischemic stroke events: systematic review and dose-response meta-analysis for the global burden of disease study 2013. BMJ 2016;354:i3857.

4 Lee I-M, Shiroma EJ, Lobelo F, et al. Effect of physical inactivity on major non-communicable diseases worldwide: an analysis of burden of disease and life expectancy. Lancet 2012;380:219-29.

5 Arem $\mathrm{H}$, Moore SC, Patel A, et al. Leisure time physical activity and mortality: a detailed pooled analysis of the dose-response relationship. JAMA Intern Med 2015;175:959-67.

6 Lin X, Zhang X, Guo J, et al. Effects of exercise training on cardiorespiratory fitness and biomarkers of cardiometabolic health: a systematic review and Meta-Analysis of randomized controlled trials. $J$ Am Heart Assoc 2015;4.

72018 Physical Activity Guidelines Advisory Committee. Physical activity guidelines Advisory Committee scientific report. Washington DC: U.S. Department of Health and Human Services, 2018.

8 Armstrong MEG, Cairns BJ, Green J, et al. Reported frequency of physical activity in a large epidemiological study: relationship to specific activities and repeatability over time. BMC Med Res Methodol 2011;11:97.

9 Reiner M, Niermann C, Jekauc D, et al. Long-term health benefits of physical activity-a systematic review of longitudinal studies. BMC Public Health 2013;13:813.

10 Kivimäki M, Lawlor DA, Smith GD, Davey Smith G, et al. Socioeconomic position, co-occurrence of behavior-related risk factors, and coronary heart disease: the Finnish public sector study. Am J Public Health 2007;97:874-9.

11 Leskinen T, Stenholm S, Heinonen OJ, et al. Change in physical activity and accumulation of cardiometabolic risk factors. Prev Med 2018;112:31-7.

12 Kujala UM, Kaprio J, Sarna S, et al. Relationship of leisure-time physical activity and mortality: the Finnish twin cohort. JAMA 1998;279:440-4.

13 Halonen JI, Stenholm S, Pentti J, et al. Childhood psychosocial adversity and adult neighborhood disadvantage as predictors of cardiovascular disease. Circulation 2015;132:371-9.

14 Statistics Finland. Classification of occupations 2001, 2002. Available: http://www.stat.fi/meta/luokitukset/ammatti/001-2001/ index_en.html

15 Swift DL, Johannsen NM, Lavie CJ, et al. The role of exercise and physical activity in weight loss and maintenance. Prog Cardiovasc Dis 2014;56:441-7.

16 Colberg SR, Sigal RJ, Fernhall B, et al. Exercise and type 2 diabetes: the American College of sports medicine and the American diabetes association: joint position statement. Diabetes Care 2010;33:e147-67.

17 Diaz KM, Shimbo D. Physical activity and the prevention of hypertension. Curr Hypertens Rep 2013;15:659-68.

18 Wasfy MM, Baggish AL. Exercise dose in clinical practice. Circulation 2016;133:2297-313.

19 Warburton DER, Charlesworth S, Ivey A, et al. A systematic review of the evidence for Canada's physical activity guidelines for adults. Int $J$ Behav Nutr Phys Act 2010;7:39.

20 Troiano RP, Berrigan D, Dodd KW, et al. Physical activity in the United States measured by Accelerometer. Med Sci Sports Exerc 2008;40:181-8.

21 WHO. Global 2010 recommendation on physical activity for health, 2010. Available: http://whqlibdoc.who.int/publications/2010/ 9789241599979_eng.pdf

22 Yu E, Rimm E, Qi L, et al. Diet, Lifestyle, Biomarkers, Genetic Factors, and Risk of Cardiovascular Disease in the Nurses' Health Studies. Am J Public Health 2016;106:1616-23.

23 Yu E, Malik VS, Hu FB. Cardiovascular Disease Prevention by Diet Modification. J Am Coll Cardiol 2018;72:914-26. 